\title{
A rat model of chronic syringomyelia induced by epidural compression of the lumbar spinal cord
}

\author{
Ji Yeoun Lee, MD, PhD, ${ }^{1-3}$ Shin Won Kim, MS, ${ }^{2}$ Saet Pyoul Kim, BS, ${ }^{1,2}$ Hyeonjin Kim, PhD, ${ }^{4}$ \\ Jung-Eun Cheon, MD, PhD, ${ }^{4}$ Seung-Ki Kim, MD, PhD, ${ }^{3,5}$ Sun Ha Paek, MD, PhD, ${ }^{5}$ \\ Dachling Pang, MD, FRCS(C), FRCS(Eng), ${ }^{6,7}$ and Kyu-Chang Wang, MD, PhD $2,3,5$
}

\begin{abstract}
Departments of ${ }^{1}$ Anatomy and ${ }^{2}$ Neural Development and Anomaly Laboratory, Seoul National University College of Medicine; ${ }^{3}$ Division of Pediatric Neurosurgery, ${ }^{4}$ Department of Radiology, ${ }^{5}$ Department of Neurosurgery, Seoul National University Children's Hospital and Seoul National University College of Medicine, Seoul, Korea; ${ }^{6}$ Department of Pediatric Neurosurgery, University of California, Davis; and ' $R$ Regional Center of Pediatric Neurosurgery, Oakland Medical Center, Kaiser Foundation Hospitals of Northern California, Oakland, California
\end{abstract}

\begin{abstract}
OBJECTIVE There has been no established animal model of syringomyelia associated with lumbosacral spinal lipoma. The research on the pathophysiology of syringomyelia has been focused on Chiari malformation, trauma, and inflammation. To understand the pathophysiology of syringomyelia associated with occult spinal dysraphism, a novel animal model of syringomyelia induced by chronic mechanical compression of the lumbar spinal cord was created.

METHODS The model was made by epidural injection of highly concentrated paste-like kaolin solution through windows created by partial laminectomy of L-1 and L-5 vertebrae. Behavioral outcome in terms of motor (Basso-Beattie-Bresnahan score) and urinary function was assessed serially for 12 weeks. Magnetic resonance images were obtained in some animals to confirm the formation of a syrinx and to monitor changes in its size. Immunohistochemical studies, including analysis for glial fibrillary acidic protein, NeuN, CC1, ED-1, and caspase-3, were done.

RESULTS By 12 weeks after the epidural compression procedure, syringomyelia formation was confirmed in $85 \%$ of the rats (34 of 40) on histology and/or MRI. The syrinx cavities were found rostral to the epidural compression. Motor deficit of varying degrees was seen immediately after the procedure in $28 \%$ of the rats (11 of 40 ). In 13 rats (33\%), lower urinary tract dysfunction was seen. Motor deficit improved by 5 weeks after the procedure, whereas urinary dysfunction mostly improved by 2 weeks. Five rats (13\%, 5 of 40 ) died 1 month postoperatively or later, and 3 of the 5 had developed urinary tract infection. At 12 weeks after the operation, $\mathrm{IHC}$ showed no inflammatory process, demyelination, or accelerated apoptosis in the spinal cords surrounding the syrinx cavities, similar to sham-operated animals.
\end{abstract}

CONCLUSIONS A novel experimental model for syringomyelia by epidural compression of the lumbar spinal cord has been created. The authors hope that it will serve as an important research tool to elucidate the pathogenesis of this type of syringomyelia, as well as the CSF hydrodynamics of the lumbar spinal cord.

https://thejns.org/doi/abs/10.3171/2016.9.SPINE16188

KEY WORDS syringomyelia; lumbar spinal cord; epidural compression; occult spinal dysraphism

0 YRINGOMYELIA is characterized by the presence of a fluid-filled cavity within the spinal cord, often involving the central canal. Syringomyelia in association with forms of occult spinal dysraphism, such as lumbosacral lipoma or tethered cord syndrome, has been well recognized and documented, especially with the wide use of MRI as a diagnostic tool for spinal dysraphism. Various clinical characteristics of this type of syringomyelia have been considered as distinct from those caused by Chiari malformation, trauma, or arachnoiditis.11,16 Also, there has long been controversy regarding its clinical management-whether direct manipulation of the syrinx through syringotomy is necessary.

We have recently reviewed a fairly large group of occult spinal dysraphism patients with syringomyelia, comparing syrinx features before and after untethering surgery,

ABBREVIATIONS BBB = Basso-Beattie-Bresnahan; GFAP = glial fibrillary acidic protein; $\mathrm{H}$ \& $\mathrm{E}=$ hematoxylin and eosin; $\mathrm{IHC}=$ immunohistochemical; IP = intraperitoneal; LFB = Luxol fast blue; PBS = phosphate-buffered saline; SCI = spinal cord injury.

SUBMITTED February 10, 2016. ACCEPTED September 22, 2016.

INCLUDE WHEN CITING Published online February 17, 2017; DOI: 10.3171/2016.9.SPINE16188. 
in an effort to resolve the argument regarding its clinical management strategy. ${ }^{17}$ The results showed that untethering alone, without direct manipulation of the syringomyelia, frequently leads to shrinking of the syringes and may be adequate management for these lesions. Interestingly, some patients show a transient increase in the syrinx index during the initial postoperative period in spite of symptomatic improvement, and surprisingly these re-expanded syringes tend to stabilize or even diminish in size without additional surgery. This observation is important because most surgeons would worry about retethering in the face of enlargement of the syrinx and might feel obligated to re-operate. These interesting but somewhat vexing clinical phenomena motivated us to undertake the present research into the pathogenesis of syrinx associated with occult spinal dysraphism.

The first step in studying the pathogenesis of a disease is an appropriate animal model exhibiting the disease's core features. Active research on syringomyelia has provided a wide selection of animal models. Conventional animal models of syringomyelia, mostly aiming to explain intramedullary cysts associated with Chiari malformation, were created by dilating the central canal or extracanalicular cavity of the spinal cord. ${ }^{4-7,20,27}$ Other approaches have used injections of excitotoxic amino acids to reproduce the excitotoxic component of syringomyelia. ${ }^{3,23,32}$ Animal models of posttraumatic syringomyelia have been established through various methods of inducing spinal cord injuries (SCI; weight-drop or clip compression injury). ${ }^{24,27,28}$ Based on these animal models, many hypotheses have been postulated on the pathogenesis of syringomyelia. However, laboratory research on syringomyelia associated with occult spinal dysraphism has been limited, and there is as yet no appropriate animal model.

In the present study we therefore aimed to develop a rat model of syringomyelia by epidural compression of the lumbar spinal cord, resembling the mass effect of lumbosacral lipoma. Various attempts to induce chronic, noninflammatory compression of the spinal cord were made. The rats in whom syringomyelia was successfully induced were evaluated in detail using neurobehavioral assessment, MRI, and immunohistochemical (IHC) studies.

\section{Methods}

\section{Animals}

Male Sprague Dawley rats (age 6 weeks, weight 175200 g) were obtained from the Preclinical Research Center of the Biomedical Research Institute of Seoul National University Hospital. A total of 55 animals were used in this study -50 in the experimental group, of which 40 survived after the operation, and 5 in the control group. The animals were kept in temperature-controlled rooms with a 12-hour light and 12-hour dark photoperiod and were fed with standard rat food and water. All procedures were approved by the Institutional Animal Care and Use Committee of Seoul National University Hospital.

\section{Experimental Procedure}

The experimental group consisted of 50 rats, who were anesthetized using ketamine (Virbac; $75 \mathrm{mg} / \mathrm{kg}$, intraperi- toneal [IP]) and xylazine (Bayer; $10 \mathrm{mg} / \mathrm{kg}$, IP). With the animal in the prone position, a midline skin incision was made from the lower thoracic to the sacral levels. The 5 lumbar laminae were exposed in the standard fashion. Partial laminectomies were performed at the L-1 and L-5 levels, leaving the L2-4 laminae intact. We found that leaving the posterior elements of the spine at L-2, L-3, and L-4 intact ensured substantial epidural compression. Kaolin (Sigma-Aldrich) was mixed with normal saline (concentration $1000 \mathrm{mg} / \mathrm{ml}$, amount $0.1-0.2 \mathrm{ml}$ ) and was injected into the epidural space through the L-1 laminectomy site using a 26-gauge needle. The injection was directed caudally until the paste-like kaolin solution squeezed out of the L-5 laminectomy site (Fig. 1). Animals in the control group (5 rats) were given sham operations with identical L-1 and L-5 partial laminectomies, as in the experimental group. After wound closure, all animals were allowed to recover under heated lamps. For postoperative analgesia, $0.1 \mathrm{ml}$ of meloxicam was administered, and gentamicin was given for antibiotic prophylaxis. Manual bladder compression was done 3 times daily until no retention of urine was noted.

\section{In Vivo Neurobehavioral Evaluation}

The motor power of the lower limbs was evaluated using the Basso-Beattie-Bresnahan (BBB) hindlimb locomotor test, ${ }^{2}$ which is a widely used, reliable method to measure hindlimb recovery in rats after experimentally induced SCI. Based on a scale of 0 to 21, the following attributes were observed and scored (maximum of 7 points each): the movement of 3 joints of the hindlimb; toe clearance, paw position, and forelimb-hindlimb coordination; trunk stability and tail position. Neurological assessment was performed every 2 days for the 1st week, then once a week until the end of the study.

The functional status of the bladder was assessed daily and classified as dysfunctional or normal. Urinary dysfunction was defined as when manual compression of the bladder was needed for complete emptying.

\section{MRI}

Rats (6 experimental rats and 1 sham-operated rat) underwent MRI 3 months after surgery. One of the 6 experimental rats underwent serial imaging, with MRI studies performed 1 month, 2 months, and 3 months postoperatively. During scanning, $1.5 \%$ isoflurane (Baxter) in room air was given for anesthesia, and the animals' respiration and temperature were monitored carefully.

All MRI data were acquired using a 9.4-T animal MR scanner (Agilent 9.4T/160AS; Agilent Technologies) with a volume coil for both radiofrequency transmission and signal reception (Agilent Technologies). Rats were positioned prone in the handling system. Following a global shimming over the entire animal body, scout images were acquired in all 3 planes using a gradient echo sequence with the following imaging parameters: TR $26 \mathrm{msec}$; TE $2.62 \mathrm{msec}$; flip angle $30^{\circ}$; number of slices, 5; slice thickness $2 \mathrm{~mm}$ without interslice gap; field of view $70 \times 70$ $\mathrm{mm}^{2}$; matrix size $128 \times 128$; receiver bandwidth $50 \mathrm{kHz}$; and 1 signal average. 

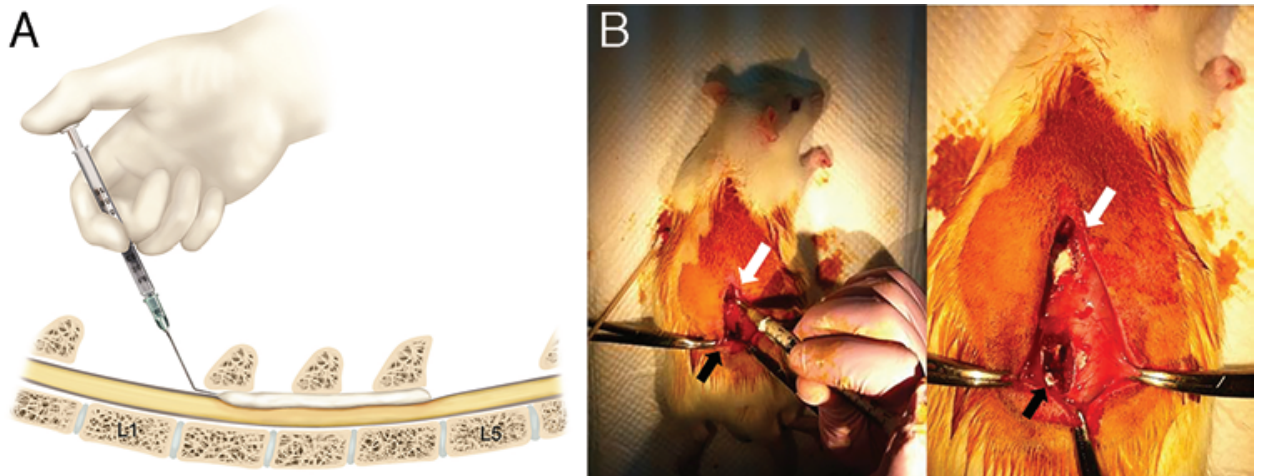

FIG. 1. Established surgical procedure. Medical illustration (A) and gross photographs (B) of epidural injection of kaolin. Injection was performed with a 1 -ml 26 -gauge syringe with the tip bent through the L-1 laminectomy site (white arrows) in the caudal direction until leakage of the kaolin was noticed in the L-5 laminectomy site (black arrows). Panel A copyright Ji Yeoun Lee. Published with permission. Figure is available in color online only.

Based on the scout image, a volume of interest (VOI) was defined followed by automated local shimming. Finally, T2-weighted images of the VOI were acquired in sagittal and/or axial planes by using a fat-saturated, respiration-gated, multiple spin-echo sequence. The imaging parameters were: TR $2000 \mathrm{msec}$; TE 13, 26, 39, 52, and 65 msec; flip angle $90^{\circ} / 180^{\circ}$; number of slices, 10 (sagittal) and 12 (axial) with interslice gap of $0 \mathrm{~mm}$ (sagittal) and 4.5-6 mm (axial); slice thickness $1 \mathrm{~mm}$ (sagittal) and 1.5 $\mathrm{mm}$ (axial); field of view $90 \times 55 \mathrm{~mm}^{2}$ (sagittal) and $55 \times$ $65 \mathrm{~mm}^{2}$ (axial); matrix size $256 \times 192$; receiver bandwidth $50 \mathrm{kHz}$; and 2 signal averages.

\section{Histopathology}

All surviving animals were killed for histological analysis 12 weeks after the operation. Rats were given a lethal dose of thiopental sodium (200 mg/kg, IP; Sigma) and perfused with $4 \%$ paraformaldehyde solution in 0.1-M phosphate buffer. The entire length of the spinal cords was harvested and immersed in 4\% paraformaldehyde solution for 24 hours. After fixation, the tissues were serially immersed in 10\%, 20\%, and 30\% sucrose solutions for dehydration. The specimens were embedded in paraffin using standard procedures. Histological sections of the entire spinal cord were made in the axial plane at 4- $\mu \mathrm{m}$ thickness and stained with hematoxylin and eosin $(\mathrm{H} \& \mathrm{E})$ and Luxol fast blue (LFB).

After $\mathrm{H} \& \mathrm{E}$ staining, the axial sections were used to evaluate the morphology and size of the central canal. The central canal to spinal cord ratio was measured on the axial section where the central canal was widest (Fig. 2).

IHC studies were done (using specimens from $13 \mathrm{ka}-$ olin solution-injected animals and 2 sham-operated controls) to evaluate the degree of inflammation and demyelination. After 3 rinses in phosphate-buffered saline (PBS), sections were blocked in normal goat serum for 30 minutes and washed in PBS. Then the sections were incubated overnight with various primary antibodies as follows: mouse-monoclonal GFAP (glial fibrillary acidic protein, 1:300 dilution, Sigma) for reactive astrocytes; mouse-monoclonal ED-1 (1:200 dilution, Millipore) for macrophages or microglia; mouse-monoclonal $\mathrm{CC} 1$
(1:300 dilution, Novus Biological) for oligodendrocytes; mouse-monoclonal NeuN (1:300 dilution, Millipore) for neurons; and rabbit-polyclonal caspase-3 (1:300 dilution, Sigma) for apoptosis. Primary antibodies were diluted in PBS containing $1 \%$ normal goat serum. After rinsing with PBS, sections were incubated for 1 hour at room temperature with biotinylated anti-mouse or anti-rabbit IgG antibodies (Vector) diluted 1:1000. The secondary antibodies were detected using an avidin-biotin complex and visualized using diaminobenzidine (Sigma). The sections were washed in PBS 3 times. The slides were analyzed using light microscopy and compared with sham-operated control samples.

\section{Results}

The rate of early mortality was fairly high $(20 \%)$. Ten of the 50 rats died or were killed during the first 5 days

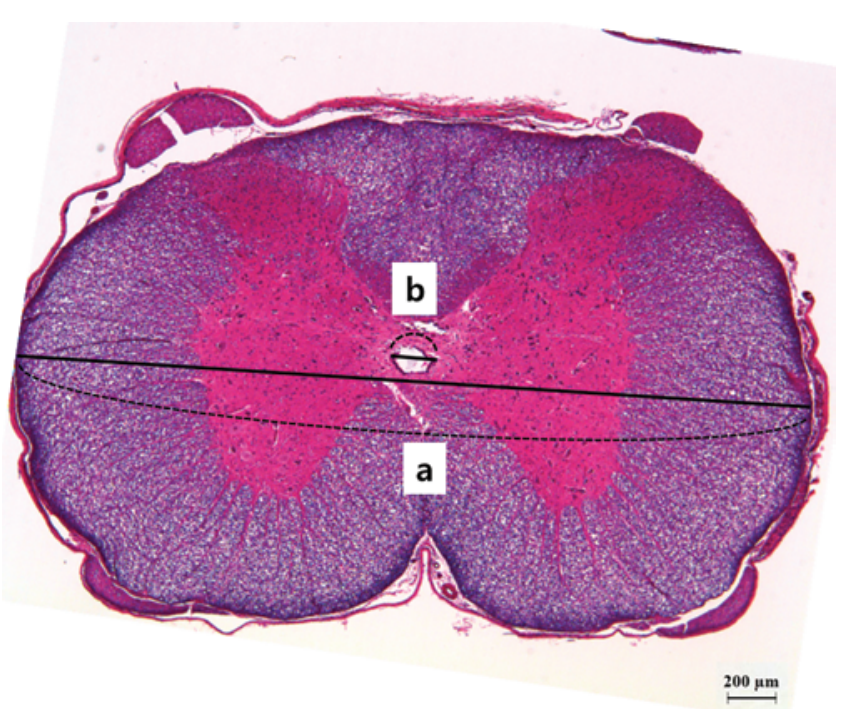

FIG. 2. Ratio of central canal to spinal cord diameter. The central canalspinal cord ratio is obtained by dividing the transverse diameter of the central canal (b) by that of the spinal cord (a) where the central canal is widest. Figure is available in color online only. 


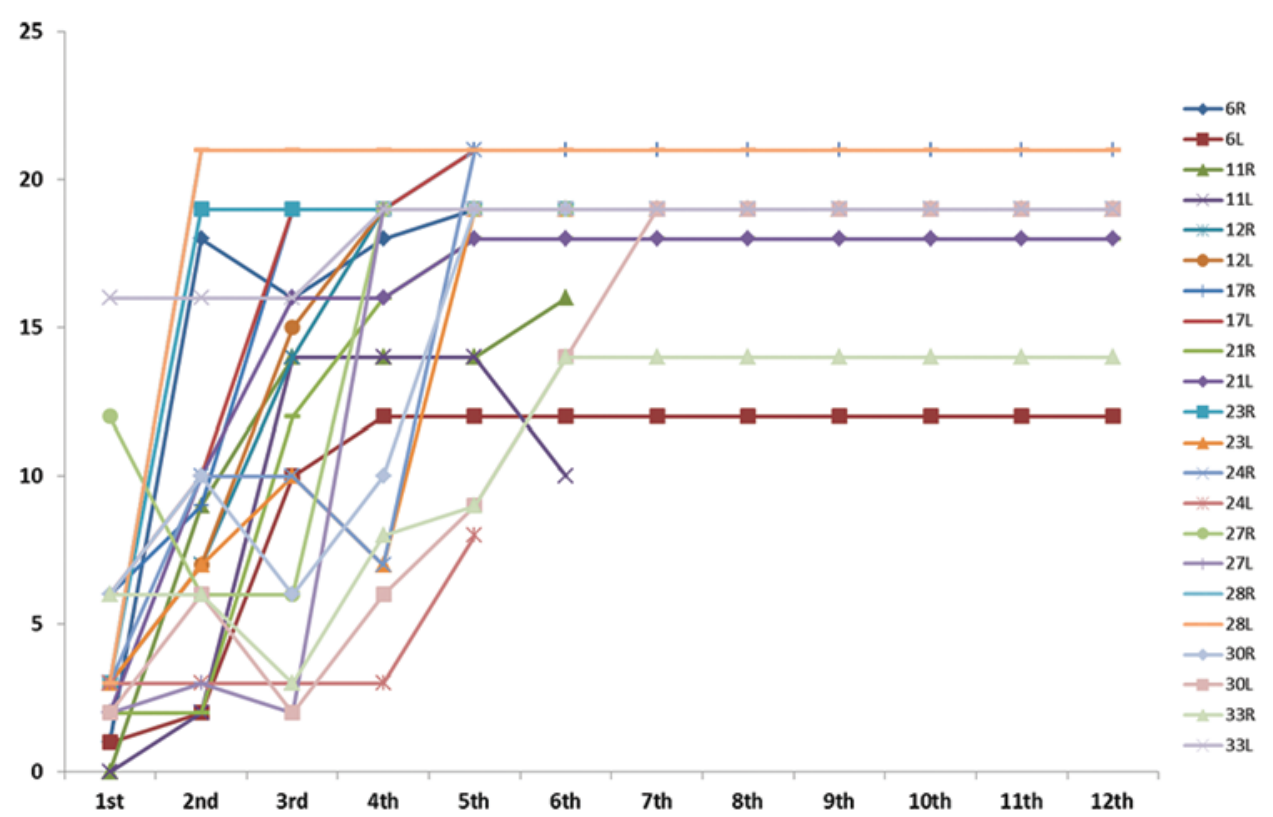

FIG. 3. Plot of BBB scores. The weekly BBB scores for each hindlimb of rats that showed motor deficit were plotted. Most of the graphs plateaued after the 5th week, meaning active recovery of the motor function is completed in this period. The $x$-axis indicates time in weeks. The $y$-axis indicates BBB scores. Scores are shown for right $(R)$ and left $(L)$ hindlimbs of rats identified by number. Figure is available in color online only.

after the operation despite analgesics and antibiotic treatment. Poor recovery from anesthesia, surgical bleeding, and severe urinary retention (full bladder with turbid or bloody urine) were probable causes of death. Two animals had seizure and autotomy and therefore had to be killed during the immediate postoperative period.

Five rats died during the subacute or delayed postoperative period (a month or more after surgery). The cause of death for 3 was suspected to be urinary tract infection due to persistent or new-onset urinary retention.

Thirty-four rats were confirmed to show syringomyelia on histology and/or MRI, resulting in a success rate of $68 \%$ (34 of 50 rats) for the group overall. Excluding the 10 early mortality cases, this model succeeded in producing syringomyelia in $85 \%$ (34 of 40) of the animals.

The sham-operated group $(n=5)$ had no mortality and stayed neurologically asymptomatic after the operation.

\section{Neurobehavioral Outcome}

Behavioral testing was done on the 40 rats that survived past the early postoperative period. Eleven of these rats $(28 \%)$ showed varying degrees of hindlimb weakness (BBB score range $0-18$ ) immediately after the operation. Two of the 11 rats died (one at 5 weeks and one at 6 weeks after the operation) due to urinary retention in spite of improving hindlimb weakness. Improvement of motor weakness was observed for 5 weeks and then plateaued (Fig. 3 ). In 5 rats, the degree of weakness in the hindlimbs was not symmetrical. This asymmetry persisted in 3 of the 5 animals. The sham-operated animals showed no change from preoperative BBB scores.

Urinary retention was noted immediately after surgery in 13 of the 40 surviving rats in the experimental group
(33\%). Although most of the 13 only had mild retention, 1 rat showed severe detrusor-sphincter dyssynergia. Urinary function fully recovered within 2 weeks in 8 animals (62\%), but in $5(38 \%)$ recovery took up to 1 month. Of the 5 rats with late recovery, one eventually died of urinary tract infection.

Correlation between delayed mortality (excluding early postoperative mortality) and neurological status was assessed. Delayed mortality was far more frequent in rats with delayed urinary dysfunction $(\mathrm{p}=0.009$, Table 1$)$.

\section{MRI}

In 5 of the 6 rats (excluding the sham-operated case) examined with MRI 3 months after surgery, the studies showed syringomyelia. In the sixth rat, there was no

TABLE 1. Correlation between delayed mortality and neurological status

\begin{tabular}{llrl}
\hline \multirow{2}{*}{ Variable } & \multicolumn{2}{c}{ Delayed Mortality } & $\begin{array}{c}p \\
\text { Value }\end{array}$ \\
\cline { 2 - 3 } & \multicolumn{1}{c}{ Yes } & No & \\
\hline Initial urinary dysfunction & & 12 & 0.469 \\
\hline Yes & 1 & 23 & \\
\hline No & 4 & & \\
\hline Initial motor deficit & & 9 & 0.422 \\
\hline Yes & 2 & 26 & \\
\hline No & 3 & & \\
\hline Delayed urinary dysfunction & & 0 & 0.009 \\
\hline Yes & 3 & 35 & \\
\hline No & 2 &
\end{tabular}



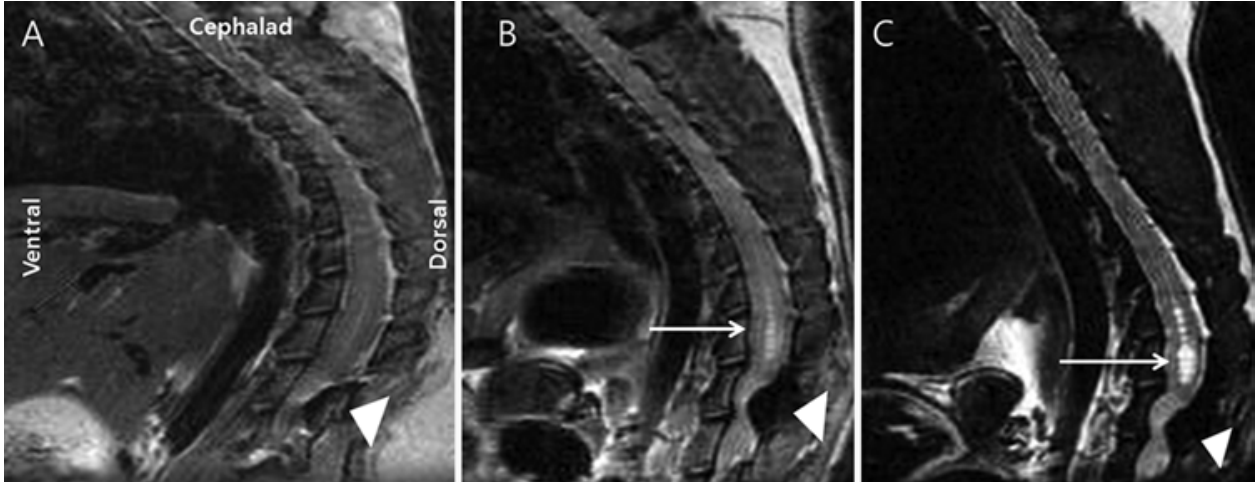

FIG. 4. Syringomyelia in a rat: serial MR images. Serial sagittal T2-weighted images obtained 1 month (A), 2 months (B), and 3 months (C) after the operation showing the appearance of syringomyelia (arrow) at 2 months cephalad to the compression site (arrowhead). Note obvious enlargement of the syrinx (arrow) at 3 months.

MRI evidence of syringomyelia. In the serial images of 1 rat, enlargement of the syrinx cavity was evident from 2 months (Fig. 4). In line with the histological data, the largest diameter of the syrinx cavity was observed just cephalad to the compression site (Figs. 4 and 5).

Whereas all the rats with prominent syringomyelia on MRI had significant compression by the injected kaolin solution, the rat without syringomyelia had minimal compression, similar to the animal that underwent sham operation (Fig. 6).

\section{Immunohistochemistry}

At 12 weeks, 34 (85\%) of 40 rats showed evidence of an intramedullary cyst rostral to the epidural compression. All cysts were distention of the central canal (true hydromyelia); no paracentral cyst in the parenchyma was noted
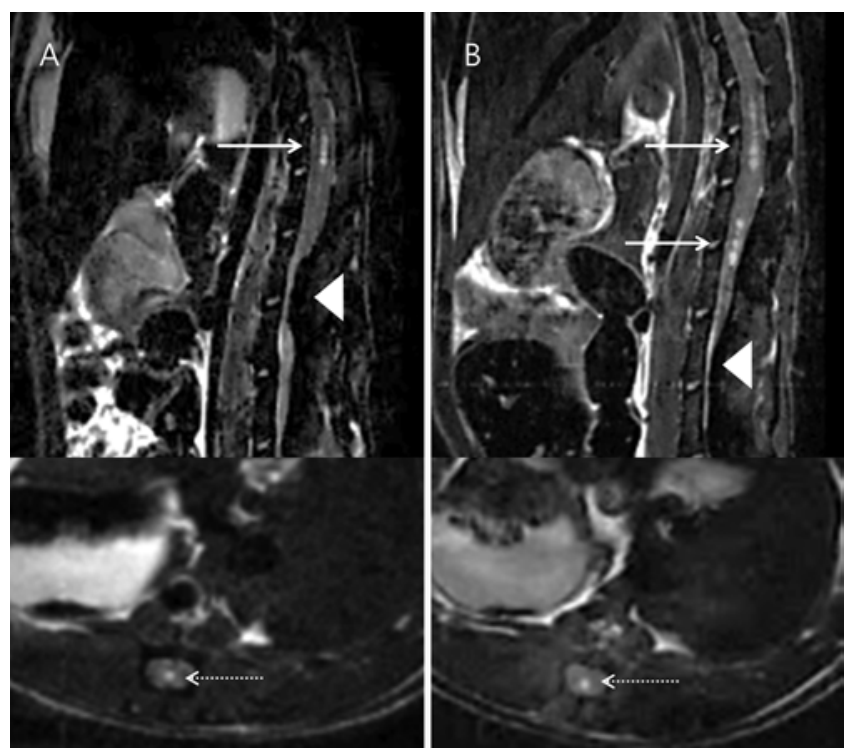

FIG. 5. Syringomyelia in a rat: MR images obtained 3 months after injection of kaolin (A). Another example of syringomyelia shown at 3 months after the operation (B) on sagittal (upper, arrows) and axial (lower, dotted arrow) T2-weighted images. The syrinx cavity is located cephalad to the compression site (arrowhead).
(Fig. 7). The ependymal lining of the central canal was intact. The average central canal to spinal cord ratio of the experimental group was 0.071 (SD 0.017), whereas it was 0.008 (SD 0.002) in the control group $(\mathrm{p}<0.05)$. A few small cystic changes in the gray matter were seen in the experimental group; such changes were not present in the control animals.

There was no significant increase in GFAP-immunoreactive astrocytes within the spinal cords of the experimental animals compared with the sham-operated ones (Fig. 8B). NeuN staining showed similar numbers and distribution of neurons in the gray matter of the 2 groups (Fig. $8 \mathrm{C})$. Also, no difference in the number of oligodendrocytes was seen between groups (Fig. 8D). Likewise, there was no evidence of macrophage infiltration or activation of resident CNS microglia in the gray matter of experimental animals as shown in the ED1 staining (Fig. 8E). A slight increase of ED1-positive cells in the dorsal white matter was seen in some specimens, but the trend was not significant. No increase of apoptotic cell death as indicated
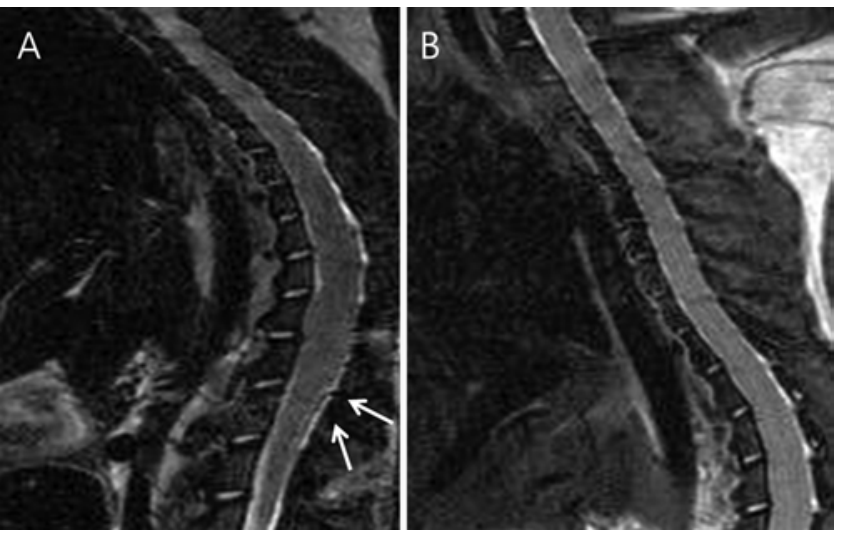

FIG. 6. Rats with no syringomyelia on MRI. Sagittal T2-weighted images obtained in the kaolin-injected rat that had no MRI evidence of syringomyelia (A) and a sham-operated rat (B). The kaolin-injected rat that did not show any syringomyelia (injected kaolin denoted by arrows) had only minimal cord compression, not too different from the sham-operated animal, suggesting that effective compression may be critical in the formation of syringomyelia in this animal model. 


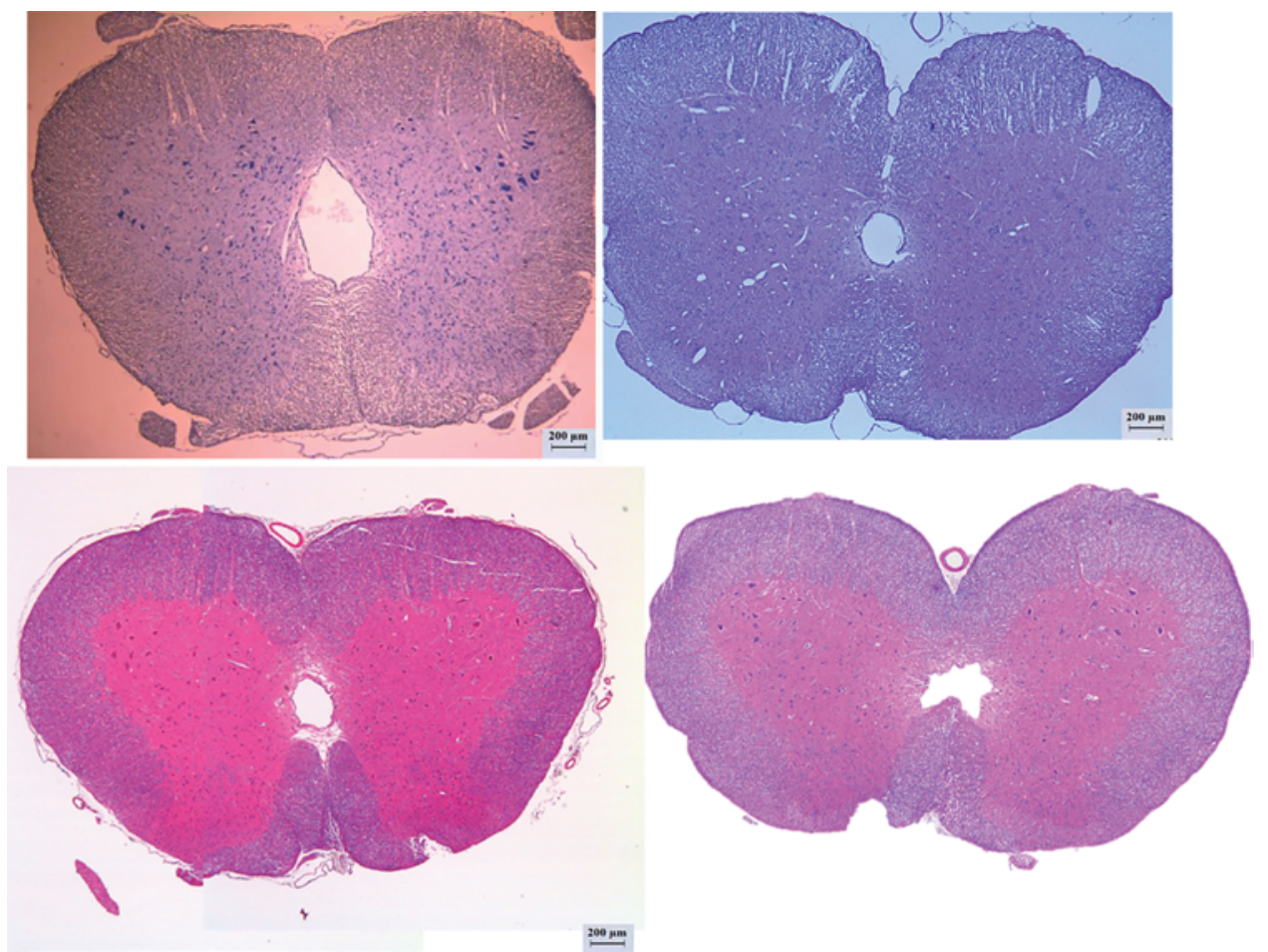

FIG. 7. Histological confirmation of syringomyelia. Axial sections of 4 representative experimental rats' spinal cords showing enlargement of the central canal. Bar $=200 \mu \mathrm{m}$. Figure is available in color online only.

by caspase-3 activity (Fig. 8F) or significant demyelination with LFB staining was noted in the syringomyelia animals compared with the controls (Fig. 8G).

We found in 2 rats that the kaolin solution had inadvertently been injected intradurally. These animals did not develop syringomyelia, but cystic changes were seen in the gray and white matter of their spinal cords (Fig. 8A). Also, there were ED1-positive macrophages near vessels of the white matter (Fig. 8E) as well as patchy demyelination (Fig. 8G). The IHC findings in the rats in the experimental group as well as those that received intradural injection of kaolin and the sham-operated rats are summarized in Table 2.

\section{Discussion}

In this study, a novel rat model of syringomyelia has been created using viscous kaolin solution to produce chronic epidural compression of the spinal cord. The epidural location for the compressive gel was chosen to avoid disturbing the intradural space in anyway.

The rate of mortality during the immediate postoperative period in this model was fairly high (20\%, 10 of 50 rats), probably from anesthesia, surgical trauma, or acute urinary retention. After 12 weeks, syringomyelia was detected in $85 \%$ of the animals (34 of 40) as evident on postmortem histological examination. The syringes were usually found cephalad to the compression site. Hindlimb motor dysfunction was seen in $11(28 \%)$ of 40 rats, and lower urinary tract dysfunction in $13(33 \%)$ of 40 . Histological examination of the spinal cords with syringomyelia uniformly showed dilation of the central canal without a single case of paracentral intramedullary cyst. IHC analysis revealed no evidence of inflammation or demyelination, unlikely etiologies of this experimental syringomyelia.

\section{Comparison With Other Noncommunicating Syringomyelia Models}

This model is a noncommunicating type of syringomyelia because the syrinx cavity is not connected to the fourth ventricle, but it seems to be unique compared with other noncommunicating syringomyelia models. First, in previous models for noncommunicating syringomyelia, inflammation was one of the main mechanisms of syrinx formation. ${ }^{19,20,31}$ In one study, subarachnoid injection of low-concentration kaolin solution in the lumbar spine of rats led to infiltration of reactive lymphocytes and formation of syringomyelia unrelated to the central canal. ${ }^{29}$ The authors concluded that the "syringomyelia" resulted from intramedullary degeneration caused by arachnoiditis. Despite the similarity in the spinal level of the lesion and use of kaolin, their model is different from ours in that extensive white matter damage, lymphocyte infiltration, and uninvolvement of the intact central canal were not found in any of our animals. A recent model combining trauma and arachnoiditis also revealed extensive macrophage/microglia-associated inflammation and astrogliosis surrounding the syrinx. ${ }^{24}$

Two of our experimental animals had inadvertent intradural injection of kaolin solution. Both showed far more ED1-positive cells in the white matter and more pronounced demyelination; spongy changes in the white matter were also profound. None of the above was seen in 
the epidural injection group. This "internal" comparison between the epidural and intradural injection groups implies that pure epidural compression in our experimental condition does not cause parenchymal changes in the cord.

Second, it should be noted that the present model exclusively causes dilation of the central canal and not cyst formation in the parenchyma-i.e., the model produces pure hydromyelias. This is different from most of the noncommunicating syringomyelia models which produce hydromyelia as well as intramedullary syringomyelia.13,20,24

Last, the timing of syrinx formation is delayed in the present model, thereby making it a "chronic" model of syringomyelia. The other noncommunicating syringomyelia models generate "acute" syringes within several days.

\section{Comparison With Other Spinal Cord Compression Models}

There have been other models of spinal cord compression, such as with tumor cell implantation, ${ }^{8,18}$ tightening of screws, ${ }^{1,26}$ insertion of plastic sheets ${ }^{12}$ or rubber, ${ }^{25}$ and inflation of balloons. ${ }^{15}$ These models may be categorized by the rate of onset and the duration of the compression. The tumor cell implantation model causes slowly escalating compression because it takes 10-20 days for the growing tumor to achieve critical size. In contrast, the balloon inflation model causes rapid and acute compression. The present model causes a less abrupt or "subacute" onset of compression because the kaolin solution that is used changes from paste to solid within an hour.

Regarding the duration of compression, previous models of balloon inflations or plastic sheet insertions sustain the compression for only a few hours to not more than a few days. More prolonged or chronic compression up to 25 weeks has been used in more recent studies. The present model, with 12 weeks of compression, may also be categorized as chronic. However, it should be noted that none of the previous compression models produced syringomyelia, regardless of speed of onset and chronicity of compression.

One recent rat model constructed to study cervical myelopathy used thin sheets of expanding polymer to cause sustained epidural compression at the C-5 and C-6 levels. ${ }^{12}$ The rats were observed for up to 25 weeks with no evidence of inflammation or demyelination as in our animals, but none had syringomyelia in the cervical model. The reason for this key difference from our model may be the location of the compression, i.e., cervical versus lumbar. The fluid dynamics within the spinal canal may be very different in various regions of the spinal column, and local compression presumably may result in region-specific cerebrospinal fluid flow disturbances. Also, whereas rats aged 5-6 weeks were used in the present study, the cervical model used 12- to 14-week-old rats, and the age difference may also influence fluid dynamics of the spinal cord. Further studies are needed to elucidate the mechanism.

\section{Delayed Neurological Deficits in the Present Model}

Although ideally we would compare our results with results from other syringomyelia models, motor and lower urinary tract dysfunction have not been analyzed in detail in previous syringomyelia models, and we therefore compared our results with those derived from SCI models.
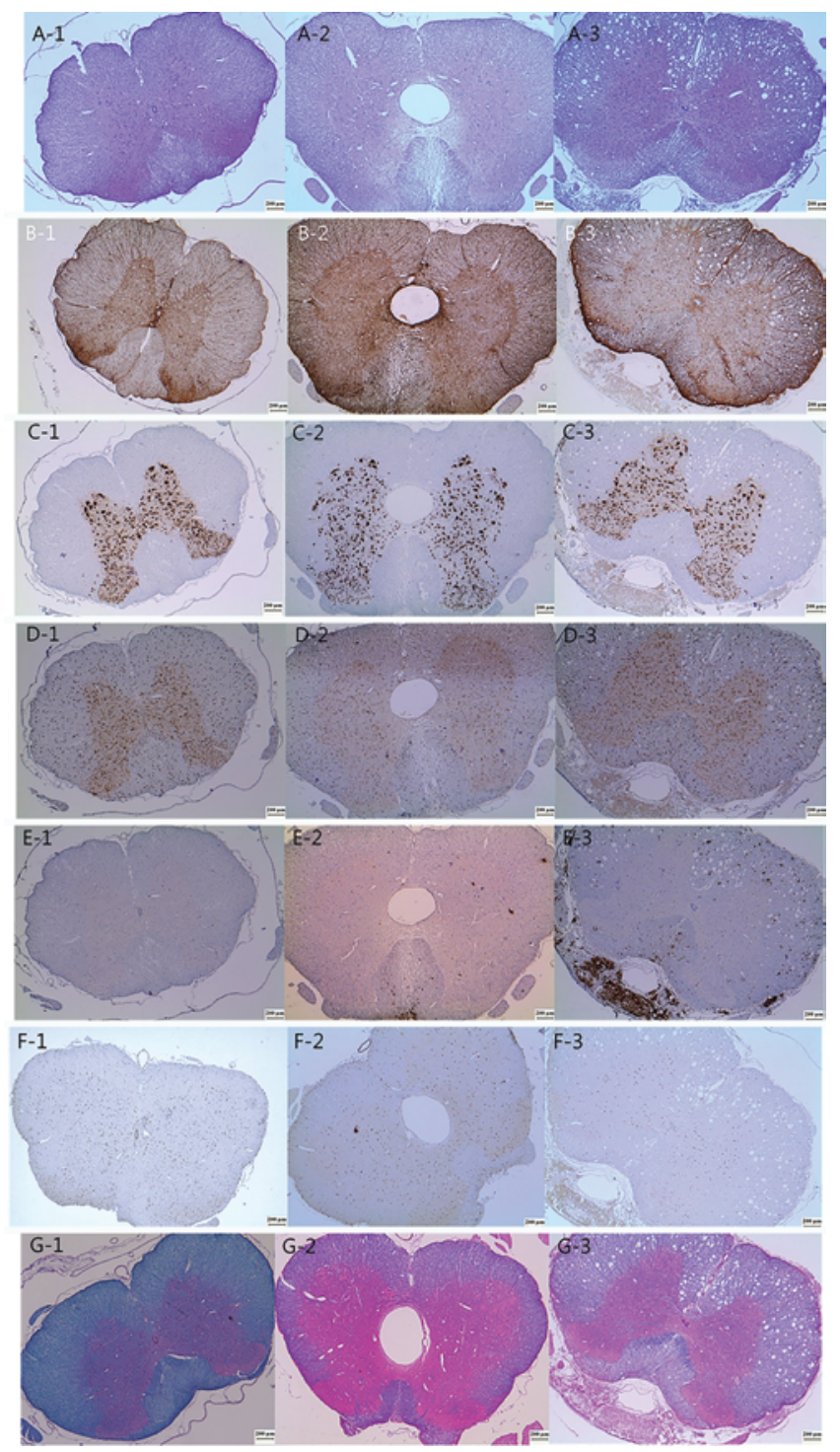

FIG. 8. Comparison of IHC results between animals that received epidural injection of kaolin, intradural injection of kaolin, and sham surgery. The first, second, and third columns are sections from rats that underwent sham surgery (A-1 to G-1), epidural injection of kaolin (A-2 to G-2), and intradural injection of kaolin (A-3 to G-3), respectively. The IHC stain for each row is as follows. A: $\mathrm{H} \& \mathrm{E}$ (morphology). B: GFAP (reactive astrocytes). C: NeuN (neurons). D: CC1 (oligodendrocytes). E: ED1 (macrophage or microglia). F: Caspase-3 (apoptosis). G: LFB (myelin). $B a r=200 \mu \mathrm{m}$. Other than the clear enlargement of the central canal (syringomyelia) seen in A-2, no difference is evident between the sham-operated group and the epidural injection group (first column, B-1 through G-1, vs second column, B-2 through G-2). H \& E staining of intradural kaolin injection (A-3) shows a small central canal but extensive spongiform changes in the dorsal and ventral white matter. Also, an increased number of ED1-positive cells are seen throughout the white matter of the animal that received intradural injection of kaolin (E-3) compared with the sham-operated rat (E-1), suggesting an inflammatory process caused by the intradural kaolin. Patchy demyelination $(G-3)$ is also observed in the animal with intradural injection. Figure is available in color online only. 
TABLE 2. Comparison between epidural injection, intradural injection, and sham groups in IHC results

\begin{tabular}{|c|c|c|c|}
\hline \multirow[b]{2}{*}{ Variable } & \multicolumn{3}{|c|}{ Group } \\
\hline & Sham & Epidural Injection (syringomyelia) & Intradural Injection \\
\hline Morphology (H \& E) & Normal & $\begin{array}{l}\text { Enlarged central canal, otherwise } \\
\text { normal }\end{array}$ & $\begin{array}{l}\text { Spongiform changes in the white } \\
\text { matter }\end{array}$ \\
\hline Extent of astrogliosis (GFAP) & $\begin{array}{l}\text { Scanty GFAP-positive astrocytes in the white } \\
\text { \& gray matter }\end{array}$ & No difference from sham & No difference from sham \\
\hline Neuronal loss (NeuN) & Neurons in the gray matter & No difference from sham & No difference from sham \\
\hline Oligodendrocytes (CC1) & Distributed throughout white \& gray matter & No difference from sham & No difference from sham \\
\hline Macrophage infiltration (ED1) & Very few & No difference from sham & Much increased in the white matter \\
\hline Apoptosis (caspase-3) & Scattered throughout the white \& gray matter & No difference from sham & No difference from sham \\
\hline Demyelination (LFB) & No demyelination & No difference from sham & Patchy demyelination \\
\hline
\end{tabular}

The animals in our model tend to have milder initial motor and bladder dysfunction, with faster and more complete recovery, than those in SCI models. ${ }^{22,24}$ The neurological deficits seen in the early postoperative period should be interpreted as symptoms due to acute surgical trauma (but to a lesser degree than in $\mathrm{SCI}$ ) rather than syringomyelia, as this model seems to be a "chronic" model of syringomyelia.

The interesting neurological trend in the study is that some of our rats suffered delayed onset of new neurological deficits. Of the rats that died unexpectedly 1 month or more after the operation, 3 had no deficits initially but developed late neurogenic bladder. Although their sudden demise precluded a new MRI and "emergent" postmortem examination of the spinal cord, progressive expansion of the syringomyelia may be speculated as one of the reasons for the delayed neurological deterioration. It is tempting to contemplate modifying this model in the future to study the long-term neurological consequences of progressive syringomyelia, but for the present study, our efforts were concentrated in producing syringomyelia.

\section{Pathogenetic Mechanism of Syringomyelia Formation}

Multiple hypotheses have been postulated to explain the genesis of syringomyelia. The oldest hypothesis is the "water-hammer theory" proposed by Gardner, followed by William's "suck effect theory" 30 and Oldfield's "piston theory," 21 but these hypotheses are focused on abnormal CSF flow dynamics at the craniovertebral junction, particularly in association with a Chiari malformation. More recently, Greitz proposed a "unified" theory of "intramedullary pulse pressure," which would provide an explanation for syringomyelia regardless of the underlying etiology. ${ }^{10}$ He stated that the syrinx is formed by fluid distension of the cord brought about by a relative increase in the pulse pressure within the central canal of the spinal cord relative to the CSF pulse pressure in the nearby subarachnoid space. According to the Bernoulli principle, the CSF pulse pressure in the subarachnoid space is decreased when the subarachnoid space is narrowed (in various situations causing partial or total obstruction of the space, such as Chiari malformation, arachnoiditis, or tumor) and subsequently the velocity of CSF flowing through the narrowed portion is increased. In our model, the kaolin material causes total obstruction of the lumbar subarachnoid space, and the result may be seen as a model of fixed and total obstruction of subarachnoid space in a fairly long segment of the spinal cord, most similar to the case of spinal tumors. However, because the CSF pulse pressure originates from the pressure wave of CSF displaced from the head during arterial pulsations, as postulated by Greitz, the compliance of the thecal sac "dampens the systolic pulse wave as it propagates down the spinal canal." 10 Therefore, in the case of spine tethering at more caudal regions, he suggests that flexion movement would be an additional cause of mechanical distension and traction of the spinal cord. Still, as discussed earlier with respect to contrasting results in syringomyelia formation in the chronic epidural compression of cervical and lumbar regions, unrevealed differences in the fluid dynamics according to the proximity to the craniocervical junction may be playing a critical role in the pathophysiology of syringomyelia in the present model. Therefore, further elucidation of the pathogenetic mechanism of the present animal model may reveal other specific elements of syringomyelia.

\section{Association With Occult Spinal Dysraphism}

Thoracolumbar syringomyelia is frequently seen in association with large lumbosacral lipomas and is occasionally seen in association with simple tethering from a thickened filum. The deleterious effect on the spinal cord for most lipomas is likely due more to the tethering than to the compression by the fat mass, and it could be beneficial for a "complete" animal model to investigate whether the traction itself independent of the mass compression could cause syrinx formation. However, constructing a tethering model in rat was technically not feasible because of the fragility of the spinal cord and filum terminale. The present model recapitulates only the compressive aspect of the pathophysiology of lumbosacral lipoma, not the role played by the tethering. In a previous model simulating cord tethering, 5.0 grams of traction was applied for 10 minutes to the filum of guinea pigs; the filum was then fixed to the sacrum with cyanoacrylate. ${ }^{14}$ Electrophysiological and morphological evaluation done 10 days after the surgical procedures revealed defective motor and sensory conduction, as well as edema and damage to the neurofilaments, axons, and myelin sheath. However, syrin- 
gomyelia was not observed, possibly because the model was an acute one.

\section{Limitations and Future Directions}

There are several limitations in this model. First, inasmuch as this model was initially designed to address the mechanisms by which lumbosacral lipomas cause syrinx formation, it only partially accomplishes its mission. Only the compressive effect on the spinal cord is simulated, not the tethering component. Furthermore, the epidural location of the compression does not quite match the intradural site of lipomas. Also, the compression was done at the most caudal portion of the spinal cord, thereby eliminating the possibility of syrinx formation caudal to the compression site. Second, the lack of correlation between progressive expansion of the syrinx and symptomatic deterioration is puzzling. This discrepancy may be artifactual, perhaps due to a lack of good assessment tools. A more thorough and quantitative assessment of urinary function and more reliable methods of sensory evaluation with longer observation periods might detect subtle changes in neurological status. The limited number of animals with MRI evaluation is another weakness in terms of our data set. Because the goal of the study was to establish a new animal model, serial imaging was also done as a "pilot" trial. In the future, we hope that more extensive imaging can be incorporated into the study design - with serial imaging being performed in more animals and possibly with all of the animals undergoing at least one postoperative MRI study. Third, our low yield of syringomyelia (68\%) is disturbing. In part this is due to the high early mortality rate of $20 \%$, which should be correctable through improvements in anesthesia and postoperative care. Also, our technique of producing adequate epidural compression could be improved, to examine cases with insufficient mass effect and consequently negative results.

Nevertheless, this study has established a novel animal model of syringomyelia caused by epidural compression of the spinal cord, resembling one aspect of the pathogenetic mechanism of lumbosacral lipomas or spinal tumors. It can serve as the platform for evaluating the pathophysiology of syringomyelia as well as the CSF hydrodynamics in the spinal canal. We hope that further studies to elucidate the possibility of new diagnostic measures and treatment modalities will be based on the model.

\section{Conclusions}

A novel animal model of syringomyelia was established by inducing epidural compression of the lumbosacral spinal cord using highly concentrated kaolin solution and allowing 12 weeks for syrinx development. The exclusive enlargement of the central canal cephalad to the compression site was observed on MRI and postmortem histological examination of the spinal cord. No evidence of inflammation in the spinal cord surrounding the syrinx was found in IHC studies, differentiating the present model from previous models of noncommunicating syringomyelia. Future studies on the pathogenetic mechanism and therapeutic strategies for the established model are being considered.

\section{Acknowledgments}

We are especially thankful to Ms. Mi Jin Jung for the medical illustration.

This research was supported by Basic Science Research Program through the National Research Foundation of Korea (NRF) funded by the Ministry of Science, ICT \& Future Planning (2015R1A2A1A15055781).

\section{References}

1. Al-Mefty O, Harkey HL, Marawi I, Haines DE, Peeler DF, Wilner HI, et al: Experimental chronic compressive cervical myelopathy. J Neurosurg 79:550-561, 1993

2. Basso DM, Beattie MS, Bresnahan JC: A sensitive and reliable locomotor rating scale for open field testing in rats. $\mathbf{J}$ Neurotrauma 12:1-21, 1995

3. Brodbelt AR, Stoodley MA, Watling A, Rogan C, Tu J, Brown CJ, et al: The role of excitotoxic injury in post-traumatic syringomyelia. J Neurotrauma 20:883-893, 2003

4. Chakrabortty S, Tamaki N, Ehara K, Ide C: Experimental syringomyelia in the rabbit: an ultrastructural study of the spinal cord tissue. Neurosurgery 35:1112-1120, 1994

5. Chang HS, Nakagawa H: Theoretical analysis of the pathophysiology of syringomyelia associated with adhesive arachnoiditis. J Neurol Neurosurg Psychiatry 75:754-757, 2004

6. Cho KH, Iwasaki Y, Imamura H, Hida K, Abe H: Experimental model of posttraumatic syringomyelia: the role of adhesive arachnoiditis in syrinx formation. J Neurosurg 80:133-139, 1994

7. Cohen WA, Young W, DeCrescito V, Horii S, Kricheff II: Posttraumatic syrinx formation: experimental study. AJNR Am J Neuroradiol 6:823-827, 1985

8. Delattre JY, Arbit E, Thaler HT, Rosenblum MK, Posner JB: A dose-response study of dexamethasone in a model of spinal cord compression caused by epidural tumor. J Neurosurg 70:920-925, 1989

9. Gardner WJ, Goodall RJ: The surgical treatment of ArnoldChiari malformation in adults; an explanation of its mechanism and importance of encephalography in diagnosis. J Neurosurg 7:199-206, 1950

10. Greitz D: Unraveling the riddle of syringomyelia. Neurosurg Rev 29:251-264, 2006

11. Iskandar BJ, Oakes WJ, McLaughlin C, Osumi AK, Tien RD: Terminal syringohydromyelia and occult spinal dysraphism. J Neurosurg 81:513-519, 1994

12. Kim P, Haisa T, Kawamoto T, Kirino T, Wakai S: Delayed myelopathy induced by chronic compression in the rat spinal cord. Ann Neurol 55:503-511, 2004

13. Kobayashi S, Kato K, Rodríguez Guerrero A, Baba H, Yoshizawa $\mathrm{H}$ : Experimental syringohydromyelia induced by adhesive arachnoiditis in the rabbit: changes in the blood-spinal cord barrier, neuroinflammatory foci, and syrinx formation. $\mathbf{J}$ Neurotrauma 29:1803-1816, 2012

14. Koçak A, Kiliç A, Nurlu G, Konan A, Kilinç K, Cirak B, et al: A new model for tethered cord syndrome: a biochemical, electrophysiological, and electron microscopic study. Pediatr Neurosurg 26:120-126, 1997

15. Konno S, Yabuki S, Sato K, Olmarker K, Kikuchi S: A model for acute, chronic, and delayed graded compression of the dog cauda equina. Presentation of the gross, microscopic, and vascular anatomy of the dog cauda equina and accuracy in pressure transmission of the compression model. Spine (Phila Pa 1976) 20:2758-2764, 1995

16. Koyanagi I, Iwasaki Y, Hida K, Abe H, Isu T, Akino M: Surgical treatment of syringomyelia associated with spinal dysraphism. Childs Nerv Syst 13:194-200, 1997

17. Lee JY, Phi JH, Cheon JE, Kim SK, Kim IO, Cho BK, et al: Preuntethering and postuntethering courses of syringomyelia 
associated with tethered spinal cord. Neurosurgery 71:2329,2012

18. Manabe S, Tanaka H, Higo Y, Park P, Ohno T, Tateishi A: Experimental analysis of the spinal cord compressed by spinal metastasis. Spine (Phila Pa 1976) 14:1308-1315, 1989

19. Milhorat TH, Adler DE, Heger IM, Miller JI, HollenbergSher JR: Histopathology of experimental hematomyelia. J Neurosurg 75:911-915, 1991

20. Milhorat TH, Nobandegani F, Miller JI, Rao C: Noncommunicating syringomyelia following occlusion of central canal in rats. Experimental model and histological findings. J Neurosurg 78:274-279, 1993

21. Oldfield EH, Muraszko K, Shawker TH, Patronas NJ: Pathophysiology of syringomyelia associated with Chiari I malformation of the cerebellar tonsils. Implications for diagnosis and treatment. J Neurosurg 80:3-15, 1994

22. Ozsoy O, Ozsoy U, Stein G, Semler O, Skouras E, Schempf $\mathrm{G}$, et al: Functional deficits and morphological changes in the neurogenic bladder match the severity of spinal cord compression. Restor Neurol Neurosci 30:363-381, 2012

23. Schwartz ED, Yezierski RP, Pattany PM, Quencer RM, Weaver RG: Diffusion-weighted MR imaging in a rat model of syringomyelia after excitotoxic spinal cord injury. AJNR Am J Neuroradiol 20:1422-1428, 1999

24. Seki T, Fehlings MG: Mechanistic insights into posttraumatic syringomyelia based on a novel in vivo animal model. Laboratory investigation. J Neurosurg Spine 8:365-375, 2008

25. Sekiguchi M, Kikuchi S, Myers RR: Experimental spinal stenosis: relationship between degree of cauda equina compression, neuropathology, and pain. Spine (Phila Pa 1976) 29:1105-1111, 2004

26. Shinomiya K, Mutoh N, Furuya K: Study of experimental cervical spondylotic myelopathy. Spine (Phila Pa 1976) 17 (10 Suppl):S383-S387, 1992

27. Stoodley MA, Gutschmidt B, Jones NR: Cerebrospinal fluid flow in an animal model of noncommunicating syringomyelia. Neurosurgery 44:1065-1076, 1999
28. Stoodley MA, Jones NR, Yang L, Brown CJ: Mechanisms underlying the formation and enlargement of noncommunicating syringomyelia: experimental studies. Neurosurg Focus 8(3):E2, 2000

29. Tamaki N, Nagashima T: [Hydrodynamics of syringomyelia.] Rinsho Shinkeigaku 35:1398-1399, 1995 (Jpn)

30. Williams B: Cerebrospinal fluid pressure changes in response to coughing. Brain 99:331-346, 1976

31. Williams B, Bentley J: Experimental communicating syringomyelia in dogs after cisternal kaolin injection. Part 1. Morphology. J Neurol Sci 48:93-107, 1980

32. Yang L, Jones NR, Stoodley MA, Blumbergs PC, Brown CJ: Excitotoxic model of post-traumatic syringomyelia in the rat. Spine (Phila Pa 1976) 26:1842-1849, 2001

\section{Disclosures}

The authors report no conflict of interest concerning the materials or methods used in this study or the findings specified in this paper.

\section{Author Contributions}

Conception and design: Wang, Lee, Paek, Pang. Acquisition of data: Lee, SW Kim, SP Kim, H Kim, Cheon. Analysis and interpretation of data: Lee, H Kim, Cheon. Drafting the article: Wang, Lee, SP Kim, H Kim, Pang. Critically revising the article: Wang, SK Kim, Paek, Pang. Reviewed submitted version of manuscript: all authors. Approved the final version of the manuscript on behalf of all authors: Wang. Study supervision: Wang.

\section{Correspondence}

Kyu-Chang Wang, Division of Pediatric Neurosurgery, Seoul National University Children's Hospital, 101 Daehakro, Jongnogu, Seoul 110-769, Republic of Korea. email: kcwang@snu.ac.kr. 